\title{
Emergent black hole dynamics in critical Floquet systems
}

\author{
Bastien Lapierre $\odot,{ }^{1,2}$ Kenny Choo, ${ }^{2}$ Clément Tauber $\odot,{ }^{1}$ Apoorv Tiwari $\odot,{ }^{2,3}$ Titus Neupert, ${ }^{2}$ and Ramasubramanian Chitra $\oplus^{1}$ \\ ${ }^{1}$ Institute for Theoretical Physics, ETH Zürich, Wolfgang-Pauli-Straße 27, 8093 Zürich, Switzerland \\ ${ }^{2}$ Department of Physics, University of Zürich, Winterthurerstraße 190, 8057 Zürich, Switzerland \\ ${ }^{3}$ Condensed Matter Theory Group, Paul Scherrer Institute, CH-5232 Villigen PSI, Switzerland
}

(Received 25 September 2019; revised manuscript received 19 February 2020; accepted 1 April 2020; published 27 April 2020)

\begin{abstract}
While driven interacting quantum matter is generically subject to heating and scrambling, certain classes of systems evade this paradigm. We study such an exceptional class in periodically driven critical $(1+1)$ dimensional systems with a spatially modulated but disorder-free time evolution operator. Instead of complete scrambling, the excitations of the system remain well defined. Their propagation is analogous to the evolution along light cones in a curved spacetime obtained by two black holes. The Hawking temperature serves as an order parameter which distinguishes between heating and nonheating phases. Beyond a timescale determined by the inverse Hawking temperature, excitations are absorbed by the black holes resulting in a singular concentration of energy at their horizon. We obtain these results analytically within conformal field theory, capitalizing on a mapping to sine-squared deformed field theories. Furthermore, by means of numerical calculations for an interacting $X X Z$ spin- $\frac{1}{2}$ chain, we demonstrate that our findings survive lattice regularization.
\end{abstract}

DOI: 10.1103/PhysRevResearch.2.023085

\section{INTRODUCTION}

Floquet quantum many-body systems provide a rich arena to explore new foundational principles of statistical physics beyond equilibrium. Interest in the field is further spurred on by experimental advances in quantum-engineered systems which permit the exploration of Floquet physics in a controlled manner $[1,2]$. The prevailing paradigm in driven systems is that closed integrable systems converge to steady states described by generalized Gibbs ensembles while interacting Floquet systems heat up to trivial infinite-temperature states where all notion of coherence is lost [3-6]. Generically, whether a system heats up or not is intimately linked to notions of integrability, ergodicity, and quantum chaos. A deeper understanding of how a system heats up due to the interplay between drive and interactions is still lacking. This may be attributed to the limited scope of analytical and numerical methods available to study such complex many-body systems.

A pioneering effort in this direction is the recently proposed Floquet conformal field theory (CFT) [7,8] (see also Ref. [9]) based on a sine-squared deformation (SSD) of a CFT. SSDs impose a specific inhomogeneous energy density profile, and were originally proposed as a numerical trick for quantum simulations [10-12]. The richness of SSDs was only recently unveiled via studies of both integrable lattice models such as quantum spin chains [13-15] and CFTs in the continuum [7,8,16-19]. It was exploited in Refs. [7,8],

Published by the American Physical Society under the terms of the Creative Commons Attribution 4.0 International license. Further distribution of this work must maintain attribution to the author(s) and the published article's title, journal citation, and DOI. in which a Floquet drive alternating between a generic CFT and its SSD analog was studied. Analyzing the entanglement entropy, an intriguing phase diagram with transitions between heating and nonheating phases was obtained.

In this paper, we address the question how does a system heat up? in this class of systems via an analytical study of dynamical two-point correlation functions in the CFT formalism and a parallel numerical study in a genuine onedimensional $X X Z$ quantum spin chain at criticality subject to the Floquet-SSD protocol. By analyzing the time evolution of two-point correlation functions, we show that the heating phase is characterized by the emergence of stroboscopic black hole horizons, which manifest as attractors at two spatial locations toward which all excitations evolve and where energy accumulates indefinitely, irrespective of initial conditions. We show that the associated Hawking temperature of the black holes serves as de facto order parameter which delineates heating and nonheating phases. The nonheating phase manifests a pseudoperiodicity in both the propagation of excitations and energy density. Furthermore, we verify the surprising robustness of the CFT prediction in both phases compared to the numerical study of the driven $X X Z$ spin chain, up to about 100 cycles. This suggests a possible experimental realization, for example with cold atoms in an optical lattice.

\section{FLOQUET-SSD DYNAMICS}

Consider a general inhomogeneous Hamiltonian on a chain of size $L$ obtained by deforming a uniform $(1+1)$ dimensional CFT:

$$
\mathcal{H}=\int_{0}^{L} d x f(x) T_{00}(x)
$$



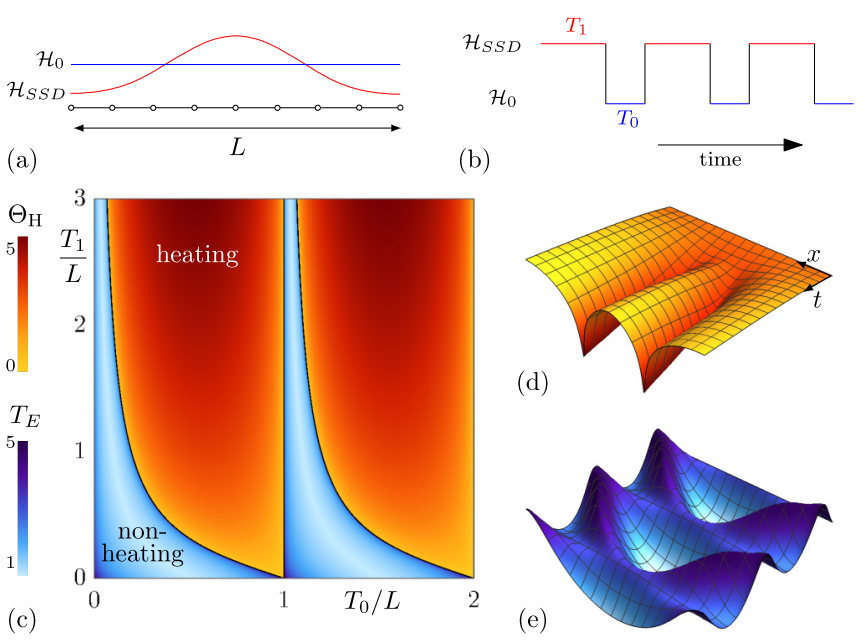

(b)

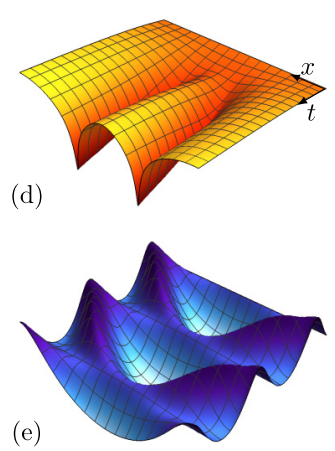

FIG. 1. (a) Uniform and SSD Hamiltonian. (b) Floquet drive alternating between the two. (c) Phase diagram (color bars in log scale). The heating phase is characterized by a Hawking temperature $\Theta_{\mathrm{H}}$, a signature of emergent black holes in the effective dynamics, whose spacetime is illustrated in (d). The nonheating phase is characterized by a pseudoperiodicity $T_{E}$, with effective spacetime illustrated in (e). The phase diagram is $\frac{T_{0}}{L}$ periodic.

We denote by $\mathcal{H}_{0}$ the homogeneous CFT where $f \equiv 1$, with energy density $T_{00}$, and by $\mathcal{H}_{\mathrm{SSD}}$ the SSD theory where $f(x)=$ $2 \sin ^{2}\left(\frac{\pi x}{L}\right)$. We consider a two-step drive protocol, where $\mathcal{H}_{\mathrm{F}}(t)$ alternates between $\mathcal{H}_{\mathrm{SSD}}$ (duration $T_{1}$ ) and $\mathcal{H}_{0}$ (duration $T_{0}$ ) as depicted in Figs. 1(a) and 1(b). The uniform theory $\mathcal{H}_{0}$ typically describes the low-energy behavior of a quantum chain at criticality and is characterized by a central charge $c$.

The lattice counterpart we explicitly consider is the $X X Z$ spin- $\frac{1}{2}$ chain,

$$
H=J \sum_{j=1}^{L-1} f_{j}\left(S_{j}^{x} S_{j+1}^{x}+S_{j}^{y} S_{j+1}^{y}+\Delta S_{j}^{z} S_{j+1}^{z}\right) .
$$

The Floquet drive $H_{\mathrm{F}}(t)$ alternates between the the uniform case, $H_{0}$ with $f_{j} \equiv 1$, and the SSD, $H_{\mathrm{SSD}}$ where $f_{j}=$ $2 \sin ^{2}\left(\frac{\pi j}{L}\right)$. For $f_{j} \equiv 1$ and $|\Delta| \leqslant 1$, the spin chain is critical and the low-energy theory is a Luttinger liquid described by a compactified free boson with $c=1$. In what follows, we will demonstrate that the general nonequilibrium exactly solvable CFT dynamics of $\mathcal{H}_{\mathrm{F}}(t)$ precisely captures the main features of the driven $X X Z$ model $H_{\mathrm{F}}(t)$ that we study numerically.

To probe the dynamics we focus on the unequal-time two-point function of the driven CFT $\mathcal{H}_{\mathrm{F}}(t), F\left(x, t ; x_{0}, 0\right) \equiv$ $\left\langle\phi(x, t) \phi\left(x_{0}, 0\right)\right\rangle$, where $\phi$ is any primary field (with conformal weight $h$ ) of the uniform theory $\mathcal{H}_{0}$ [15]. Though the full time evolution including micromotion can be evaluated, we focus on the stroboscopic evolution, where $t=$ $n\left(T_{0}+T_{1}\right), n \in \mathbb{N}$. As boundary conditions do not qualitatively affect the ensuing results, we use periodic boundary conditions for computational simplicity. Expectation values are computed in the ground state $|0\rangle$ of the uniform theory. In terms of the Virasoro generators $L_{n}$ and $\bar{L}_{n}$, in the Euclidean framework with imaginary time $\tau, \mathcal{H}_{0}=L_{0}+\bar{L}_{0}$, and crucially, $\mathcal{H}_{\mathrm{SSD}}=L_{0}-\frac{1}{2}\left(L_{1}+L_{-1}\right)+\bar{L}_{0}-\frac{1}{2}\left(\bar{L}_{1}+\bar{L}_{-1}\right)$. Such a Hamiltonian is equivalent to a uniform $\mathcal{H}_{0}$ up to an asymptotic $S L(2, \mathbb{R})$ transformation [7,16,17]. Consequently, time evolution $e^{-\tau \mathcal{H}_{\mathrm{SSD}}}$ is a simple dilation up to a coordinate change. Mapping the coordinates $w=\tau+i x$ on the cylinder to the complex plane spanned by $z=e^{2 \pi w / L}$, the coordinate change for a 1-cycle time evolution is explicitly given by

$$
\tilde{z}_{1}=\frac{\left(1+\frac{\pi \tau_{1}}{L}\right) e^{\frac{\pi \tau_{0}}{L}} z-\frac{\pi \tau_{1}}{L} e^{-\frac{\pi \tau_{0}}{L}}}{\frac{\pi \tau_{1}}{L} e^{\frac{\pi 0_{0}}{L}} z+\left(1-\frac{\pi \tau_{1}}{L}\right) e^{-\frac{\pi \tau_{0}}{L}}}=: \frac{a z+b}{c z+d} .
$$

Similarly, the time evolution after $n$ cycles is simply given by the composition of 1-cycle evolution $\tilde{z}_{n}(z)=\left(\tilde{z}_{1} \circ \ldots \circ \tilde{z}_{1}\right)(z)$. Due to the algebraic properties of the Möbius transformation it is possible to get a closed form for such a transformation, which is also a Möbius transformation:

$$
\tilde{z}_{n}=\frac{\left(\gamma_{1}-\eta^{n} \gamma_{2}\right) z+\left(\eta^{n}-1\right) \gamma_{1} \gamma_{2}}{\left(1-\eta^{n}\right) z+\gamma_{1} \eta^{n}-\gamma_{2}},
$$

where $n$ is the number of drive cycles, $\eta$ is the so-called multiplier of the 1-cycle Möbius transformation (3), and $\gamma_{1}, \gamma_{2}$ are the fixed points of this transformation. These are complex parameters that depend on $\frac{T_{0}}{L}$ and $\frac{T_{1}}{L}$, explicitly given by

$$
\begin{gathered}
\gamma_{1}=\frac{a-d-\sqrt{(a-d)^{2}+4 b c}}{2 c}, \\
\gamma_{2}=\frac{a-d+\sqrt{(a-d)^{2}+4 b c}}{2 c}, \\
\eta=\frac{a+d+\sqrt{(a-d)^{2}+4 b c}}{a+d-\sqrt{(a-d)^{2}+4 b c}} .
\end{gathered}
$$

$F\left(x, t ; x_{0}, 0\right)$ can be directly computed in the transformed coordinates, leading to the final result:

$$
\left\langle\phi(x, t) \phi\left(x_{0}, 0\right)\right\rangle=\left[\left(\frac{2 \pi}{L}\right)^{4} \frac{\partial \tilde{z}_{n}}{\partial z} \frac{\partial \overline{\tilde{z}}_{n}}{\partial \bar{z}}\right]^{h}\left\langle\phi\left(\tilde{z}_{n}, \overline{\tilde{z}}_{n}\right) \phi\left(\tilde{z}_{0}, \overline{\tilde{z}}_{0}\right)\right\rangle,
$$

where the two-point function on the right-hand side corresponds to the one evaluated in the uniform CFT, namely $\left\langle\phi\left(\tilde{z}_{n}, \overline{\tilde{z}}_{n}\right) \phi\left(\tilde{z}_{0}, \overline{\tilde{z}}_{0}\right)\right\rangle=\left(\tilde{z}_{n}-\tilde{z}_{0}\right)^{-2 h}\left(\overline{\tilde{z}}_{n}-\overline{\tilde{z}}_{0}\right)^{-2 h}$. Remarkably, the nontrivial Floquet dynamics is fully encoded in Möbius transformation $\tilde{z}_{n}$ [8]. This result is valid for a generic CFT. The central charge enters only via the conformal dimensions of the operators in the correlation function. Moreover, although Eq. (6) captures the stroboscopic dynamics of $\mathcal{H}_{\mathrm{F}}(t)$, it is well defined not only at discrete but at all continuous times-a fact that we will exploit below. We now discuss the two distinct regimes of behavior classified by the parameter $\eta$ : (i) a heating phase for $\eta \in \mathbb{R}^{+}$, and (ii) a nonheating phase with $\eta \in \mathbb{C},|\eta|=1$, with $\eta=1$ signaling the transition between the two. Equivalently, the phases are classified by the different types of 1-cycle Möbius transformation (3): elliptic for the nonheating phase, hyperbolic for the heating phase, and parabolic at the phase transition [8]. The corresponding phase diagram is given in Fig. 1(c).

\section{EMERGENT SPACETIME PERSPECTIVE ON HEATING}

The two-point function at different times $F\left(x, t ; x_{0}, 0\right)$ enables one to access the light-cone propagation of the gapless 
excitations in the system. For homogeneous Luttinger liquids, the excitations follow straight lines in spacetime. However for a class of inhomogeneous Luttinger liquids with spatial deformation $v(x)$, they follow curves, which are simply lightlike geodesics in an effective curved spacetime specified by the metric $d s^{2}=d x^{2}+v(x)^{2} d \tau^{2}$ as shown in Ref. [15]. The aim of this section is to derive the effective curved spacetime for the Floquet drive at stroboscopic times. The strategy is to find coordinates in which the effective metric describing the $n$-cycle Floquet drive is conformally flat, and then transform back to the original coordinates $(x, \tau)$. Such coordinates are called isothermal coordinates and always exist in $(1+1)$ dimensional spacetimes. Note that the calculation of the twopoint function (6) is reduced to a uniform CFT computation in the variable $\tilde{z}_{n}$. Therefore this should define isothermal coordinates, namely, $d \tilde{z}_{n} d \overline{\tilde{z}}_{n}=e^{2 \sigma(x, \tau)} d s^{2}$, where $\tilde{z}_{n}$ is the Möbius transformation Eq. (4), and $e^{2 \sigma(x, \tau)}$ is a conformal factor given by

$$
e^{2 \sigma(x, \tau)}=\left(\frac{2 \pi}{L}\right)^{2} \frac{\partial \tilde{z}_{n}}{\partial z} \frac{\partial \overline{\tilde{z}}_{n}}{\partial \bar{z}} .
$$

Therefore the change of coordinates can be computed explicitly. After analytic continuation to real time, one obtains

$$
d s^{2}=d x^{2}-g(x) d t^{2}+2 h(x) d x d t,
$$

in the original coordinates. Here, $g$ and $h$ are time-independent real functions, explicitly given in Appendix B. Therefore, the Floquet drive is equivalent to a free propagation in a stationary curved spacetime. The two-point function can be computed directly from the curved-spacetime formalism using $[7,20]$

$$
\left\langle\phi(x, \tau) \phi\left(x_{0}, 0\right)\right\rangle=e^{2 h \sigma(x, \tau)}\left\langle\phi\left(\tilde{z}_{n}, \overline{\tilde{z}}_{n}\right) \phi\left(\tilde{z}_{0}, \overline{\tilde{z}}_{0}\right)\right\rangle_{\text {flat }},
$$

where $\left\langle\phi\left(\tilde{z}_{n}, \overline{\tilde{z}}_{n}\right) \phi\left(\tilde{z}_{0}, \overline{\tilde{z}}_{0}\right)\right\rangle_{\text {flat }}$ is the usual two-point correlator in flat spacetime $d \tilde{z}_{n} d \overline{\tilde{z}}_{n}$. Using Eq. (7), this curved-spacetime approach also yields Eq. (6) up to an extra $\left(\frac{2 \pi}{L}\right)^{2 h}$ factor coming from $e^{2 h \sigma\left(x_{0}, 0\right)}$.

The null geodesics determined by the light-like curves $d s^{2}=0$ can be computed for such a metric and lead to a nonuniform velocity $v(x)=h(x) \pm \sqrt{h(x)^{2}+g(x)}$, where \pm stands for the chiral and antichiral excitations, respectively. Finally, by shifting the Floquet drive protocol by a half-period, one may obtain a time-reversal-symmetric drive such that the $d x d t$ term in Eq. (8) vanishes, implying that the fixed points are inverse of each other $\gamma_{2}=\gamma_{1}^{-1}$. Therefore the associated curved spacetime is static $d s^{2}=d x^{2}-v(x)^{2} d t^{2}$, with

$$
v(x)=\frac{1}{2 \pi i} \frac{L \ln (\eta)}{\left(T_{0}+T_{1}\right)} \frac{\left(1+\gamma_{1}^{2}-2 \gamma_{1} \cos \frac{2 \pi x}{L}\right)}{\gamma_{1}^{2}-1} .
$$

Heating phase. In this regime, $\eta \in \mathbb{R}^{+}$, in which case $\tilde{z}_{n} \rightarrow \gamma_{1}$ or $\gamma_{2}$ as $n \rightarrow \infty$ (depending on the sign of $\eta-1$ ). A typical two-point function is plotted in Fig. 2(a). After an initial transient regime during which excitations move quasiballistically, the correlation function at stroboscopic times tends to aggregate at two symmetric spatial locations denoted by $x_{c}$ and $L-x_{c}$, independently of the initial condition $x_{0}$, and only dependent on the parameters of the drive $\frac{T_{0}}{L}$ and $\frac{T_{1}}{L}$. Furthermore, the magnitude of the two-point function grows with time indicating that the excitations accumulate indefinitely at the two "horizons." This phenomenon can be interpreted in
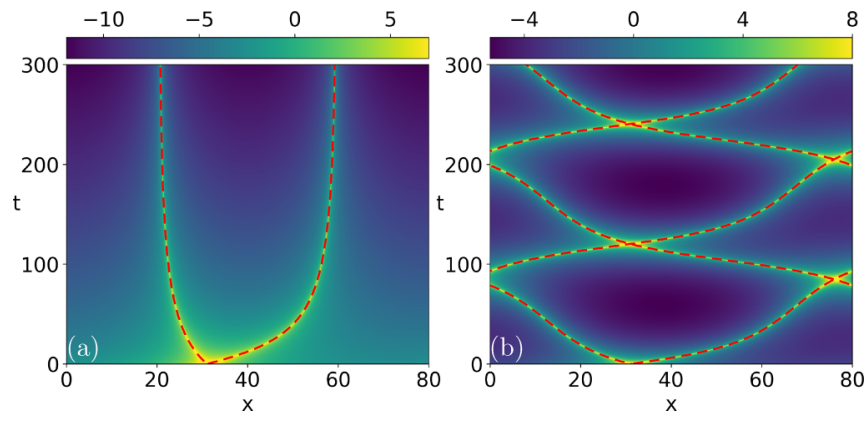

FIG. 2. CFT two-point function $\left|\left\langle\phi(x, t) \phi\left(x_{0}, 0\right)\right\rangle\right|$ for the Floquet drive $\left(L=80, x_{0}=31\right.$; color bars in log scale). (a) Heating phase $\left(T_{0}=T_{1}=34\right)$. The excitations are attracted by two black hole horizons at $x_{c}$ and $L-x_{c}$. (b) Nonheating phase $\left(T_{0}=T_{1}=25\right)$. The dynamics is pseudoperiodic. In both cases, the dashed curves are the null geodesics of the curved stationary metric.

analogy with black holes emerging from a curved spacetime, using the stationary metric (8). The corresponding trajectories fit perfectly with the analytic two-point function of Fig. 2(a). In the heating phase, $v\left(x_{c}\right)=v\left(L-x_{c}\right)=0$ such that once excitations arrive at these locations, they are permanently trapped.

To explore further the analogy with black holes in this curved spacetime, we start with a time-reversal-symmetric drive. Consequently, from $v\left(x_{c}\right)=v\left(L-x_{c}\right)=0$ one infers $x_{c}=\frac{L}{2 \pi} \arccos \left(\cos \frac{\pi T_{0}}{L}+\frac{L}{\pi T_{1}} \sin \frac{\pi T_{0}}{L}\right)$ and

$$
v(x)=2 A \frac{\sin \left[\frac{\pi}{L}\left(x-x_{c}\right)\right] \sin \left[\frac{\pi}{L}\left(x+x_{c}\right)\right]}{\cos \left(\frac{2 \pi x_{c}}{L}\right)},
$$

with $A=\frac{1+\gamma_{1}^{2}}{\gamma_{1}^{2}-1} \frac{L \ln \eta}{2 \pi i\left(T_{0}+T_{1}\right)} \in \mathbb{R}$. Near the horizon $x_{c}$, one finds that at leading order in $x-x_{c}$,

$$
d s^{2}=-C^{2}\left(x-x_{c}\right)^{2} d t^{2}+d x^{2},
$$

with $C^{2}=4 A^{2} \tan ^{2}\left(\frac{2 \pi x_{c}}{L}\right) \frac{\pi^{2}}{L^{2}}$. This is a Rindler metric which is equivalent to a black hole metric in $(1+1)$ dimensions [21-23], up to a coordinate change $\frac{C}{2}\left(x-x_{c}\right)^{2}=\left(y-x_{c}\right)$ :

$$
d s^{2}=-2 C\left(y-x_{c}\right) d t^{2}+\frac{1}{2 C} \frac{1}{\left(y-x_{c}\right)} d y^{2} .
$$

The corresponding Hawking temperature

$$
\Theta_{\mathrm{H}}=\frac{|C|}{2 \pi}=\frac{|\ln (\eta)|}{2 \pi\left(T_{0}+T_{1}\right)}
$$

is plotted in Fig. 1(c). The inverse Hawking temperature provides a timescale after which the excitations are fully trapped at $x=x_{c}$. This timescale diverges at the transition to the nonheating phase, where $\Theta_{\mathrm{H}} \rightarrow 0$. A similar expansion of $f$ near $x=L-x_{c}$ leads to a similar metric with the same $\Theta_{\mathrm{H}}$.

Nonheating phase. A typical two-point function in the nonheating phase is plotted in Fig. 2(b). As expected, the excitations are coherent, resulting in an oscillatory behavior characterized by a new periodicity $T_{E}=2 \pi \frac{T_{0}+T_{1}}{|\ln (\eta)|}$, reminiscent of discrete time crystals [24,25]; see Fig. 1(c). Notice that $T_{E}$ is in general not an integer multiple of $T_{0}+T_{1}$ so it is only a pseudoperiodicity for the original dynamics. Nevertheless, for some specific values of $T_{0}$ and $T_{1}$ such that $|\ln (\eta)|=2 \pi p$ 

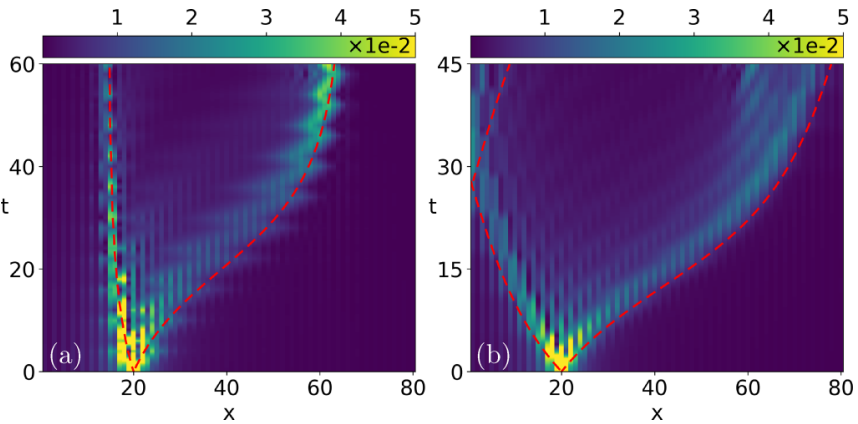

FIG. 3. $X X Z$ two-point function $\left|\left\langle S_{z}(x, t) S_{z}\left(x_{0}, 0\right)\right\rangle\right|$ for the Floquet drive $(\Delta=0.5, L=80)$. (a) Heating phase $\left(T_{0}=2, T_{1}=4\right)$. (b) Nonheating phase $\left(T_{0}=-2, T_{1}=4\right)$. The dashed curves are the null geodesics of the curved stationary metric from the CFT approach.

with $p \in \mathbb{N}, T_{E}$ is indeed an integer multiple of the underlying periodicity of the drive.

The curved-spacetime interpretation presented earlier is also valid in the nonheating phase; i.e., the excitations move ballistically in curved spacetime with the stationary metric (8). The main difference is that no horizons exist and the corresponding velocity $v$ for the null geodesics is nonzero everywhere, so the excitation can traverse the entire physical extent of the system. Our analytic results are well described by these geodesics.

\section{DRIVEN $X X Z$ MODEL}

To see whether the emergent black holes in the CFT analysis survive in a realistic condensed matter setting, we numerically simulate the $X X Z$ spin chain described in Eq. (2) subject to the Floquet-SSD driving protocol. We use matrix product states (MPS) techniques to compute the two-point spin correlation function $\left\langle G\left|S^{z}(x, t) S^{z}\left(x_{0}, 0\right)\right| G\right\rangle=$ $\left\langle G\left|U^{\dagger}(t) S^{z}(x, 0) U(t) S^{z}\left(x_{0}, 0\right)\right| G\right\rangle$ in both the heating and nonheating phase. This is done using the ITensor library [26] by simply taking the overlap between $U(t) S^{z}\left(x_{0}, 0\right)|G\rangle$ and $S^{z}(x, 0) U(t)|G\rangle$, where the unitary evolution is implemented by the sequential application of trotter gates. The numerically computed spin correlation functions are plotted in Fig. 3 and manifest a remarkably good agreement with the stroboscopic CFT predictions for $c=1$ and a compactification radius $R^{2}=$ $\frac{2(\pi-\arccos \Delta)}{\pi} . S^{z}$ corresponds to a combination of primary fields in the CFT sense. In the heating phase, we clearly see the emergence of the two predicted black hole horizons and the excitations follow the null geodesics of the curved spacetime (8). Notice that for numerical purposes we evolve with $-H_{0}$ instead of $H_{0}$, or equivalently we use a negative time $T_{0}$ , which is fine because the phase diagram of Fig. 1 is $\frac{T_{0}}{L}$ periodic. In the nonheating phase, the black hole horizons are absent and the geodesics from CFT fit the numerical data well. The effective periodicity $T_{E}$ is not seen in the simulations due to computational limitations on long-time physics.

We briefly discuss the role of micromotion within a period. We find that micromotion can indeed lead to additional interesting features both in the CFT as well as in the physical system on a lattice. Here, we focus on regimes where micromotion is reduced to small fluctuations around the stroboscopic dynamics, and can be neglected to first order. A thorough study of the role of micromotion will be addressed in future work [27].

We find that CFT provides a good description of the dynamics of the lattice system for multiple cycles of the drive. Deviations are expected to arise, when CFT overestimates the underlying entanglement entropy and total energy as a function of the number of drive cycles. This is to be expected because entropy in the finite-sized lattice is bounded by the size of the available Hilbert space, whereas in a continuum field theory such a bound does not exist and entropy can grow indefinitely. Additionally, as the energy increases beyond a threshold, one expects contributions from high-energy states not describable within CFT. Regarding the long-time behavior of the lattice system, first we note that this is hard to obtain as systematical errors grow with time. Nonetheless, in the nonheating phases, we expect the predictions of the CFT, such as oscillatory behaviors in the observables, to be qualitatively valid. In the heating phase, we expect the horizons at which the energy accumulates to still manifest. The timescales at which deviations from the CFT results arise typically depend on the parameters of the drive and can range from a few tens of cycles to hundreds of cycles. These timescales are sufficiently long, making the experimental observation of our results in a quantum-engineered setup, for example cold atoms in an optical lattice, highly feasible.

\section{ENERGY PROPAGATION}

The time evolution of the energy density $\mathcal{E}(x, t)=$ $\left\langle T_{00}(x, t)\right\rangle$ provides yet another remarkable validation of the CFT description of the Floquet-SSD $X X Z$ model. For nontrivial energy dynamics, we choose the ground state $|G\rangle$ of the open chain as the initial state, because for the periodic chain $\mathcal{E}(x, 0) \equiv 0$ in the ground state. The discussion is also applicable to other choices of initial states, such as excited states of the periodic chain. The computation of the time evolution of the energy is similar to the two-point function above, except that $T_{00}$ is not primary and boundaries cannot be neglected. Using boundary CFT techniques, we obtain

$$
\mathcal{E}(x, t)=\xi\left[\left(\frac{\partial \tilde{z}_{n}}{\partial z}\right)^{2} \frac{z^{2}}{\tilde{z}_{n}^{2}}+\left(\frac{\partial \overline{\tilde{z}}_{n}}{\partial \bar{z}}\right)^{2} \frac{\bar{z}^{2}}{\overline{\bar{z}}_{n}^{2}}\right]-\frac{\pi^{2} c}{3 L^{2}},
$$

where $\xi=\frac{c}{32}\left(\frac{2 \pi}{L}\right)^{2}, c$ is the central charge of the theory, and $\tilde{z}_{n}$ is given by Eq. (4). The details of the computation can be found in Appendix D. In Fig. 4, we show a comparison between the analytically and numerically obtained energy densities. In the nonheating phase, the energy oscillates in time with pseudoperiodicity $T_{E}$, while in the heating phase, energy accumulates indefinitely at the two horizons $x_{c}$ and $L-x_{c}$. Away from these two points, $\mathcal{E} \rightarrow 0$ as $t \rightarrow \infty$, whereas $\mathcal{E}\left(x_{c}, t\right) \sim e^{4 \pi \Theta_{\mathrm{H}} t}$. We see that heating is spatially nonuniform and occurs on the timescale $\Theta_{\mathrm{H}}^{-1}$, only defined with the parameters $T_{0}, T_{1}, L$. Finally the total energy $E(t)=$ $\int_{0}^{L} \mathcal{E}(x, t) d x$ grows exponentially in the heating phase, with a heating rate also defined by $\Theta_{\mathrm{H}}^{-1}$, and oscillates with period $T_{E}$ in the nonheating phase. 

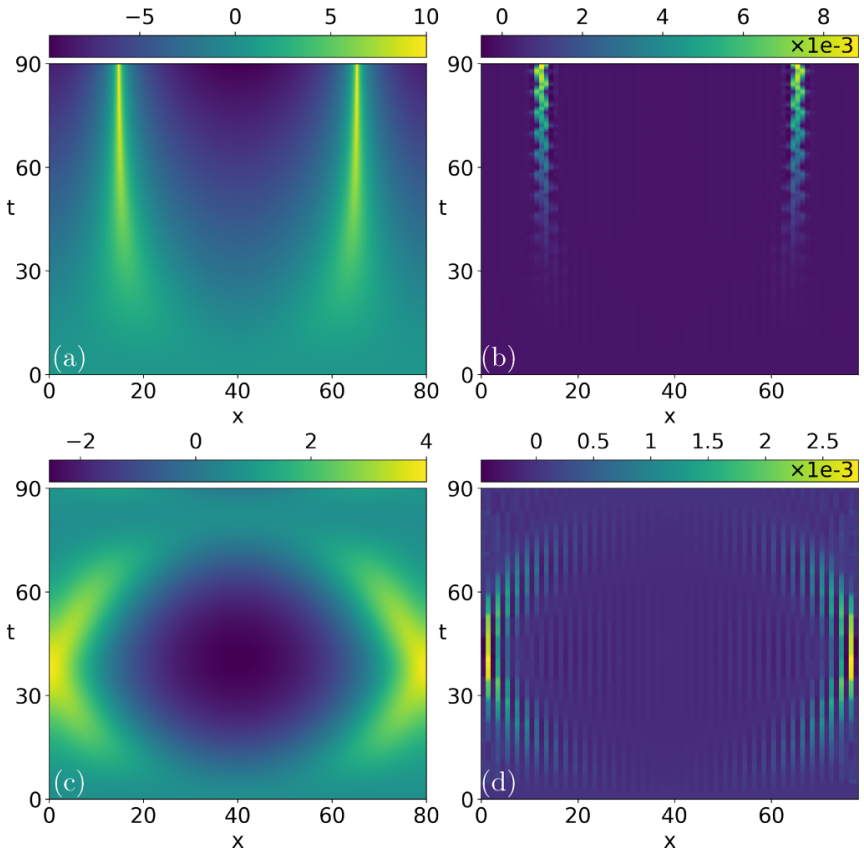

FIG. 4. Evolution of energy density $\mathcal{E}(x, t)$ in both phases, comparing CFT computation with numerical simulations $\left(L=80, T_{1}=\right.$ $4, \Delta=0.5)$. In the heating phase [(a) and (b); $T_{0}=-2$ ], the energy accumulates indefinitely at the black hole horizons with a timescale $\Theta_{\mathrm{H}}^{-1}$. In the nonheating phase [(c) and (d); $\left.T_{0}=2\right]$, the energy oscillates in the whole chain with pseudoperiodicity $T_{E}$.

\section{EFFECTIVE HAMILTONIAN}

The curved-spacetime description paradoxically suggests the existence of a well-defined Floquet effective Hamiltonian $\mathcal{H}_{\text {eff }}=\int d x v(x) T_{00}(x)$ in both phases. Note that $v$ is the velocity profile appearing in Eq. (10) (the time-reversalsymmetric case where $h=0$ ). We infer

$$
\mathcal{H}_{\text {eff }}=\alpha\left[L_{0}-\frac{\beta}{2}\left(L_{1}+L_{-1}\right)+\bar{L}_{0}-\frac{\beta}{2}\left(\bar{L}_{1}+\bar{L}_{-1}\right)\right],
$$

where $\beta^{-1}=\cos \left(\frac{\pi T_{0}}{L}\right)+\frac{L}{\pi T_{1}} \sin \left(\frac{\pi T_{0}}{L}\right)$. In the nonheating phase, $|\beta|<1$ and $\mathcal{H}_{\text {eff }}$ is related to the uniform theory $H_{0}$ by an $S L(2, \mathbb{R})$ transformation, as discussed in Appendix C. At the transition, $\beta=1$ and we recover the SSD Hamiltonian. In the heating phase, $|\beta|>1$ and the relation with $H_{0}$ instead requires $S L(2, \mathbb{C})$, leading to drastically different behavior. In particular, we find that $\mathcal{H}_{\text {eff }}$ is unbounded from below in the heating phase as can be seen from the (co)adjoint orbits of $S L(2, \mathbb{R})$ [28]. A simpler way to see this is through conformal quantum mechanics [29,30]: any Hamiltonian of the form $a L_{1}+b L_{0}+c L_{-1}$ has a classical counterpart $H=\frac{p^{2}}{2}+V(q)$ with $V(q)=\frac{1}{2} \frac{1}{q^{2}}+\frac{c^{(2)}}{8} q^{2}$ with $c^{(2)}=b^{2}-4 a c$ the quadratic Casimir invariant. In our case $c^{(2)}=\alpha^{2}\left(1-\beta^{2}\right)$ so that $V$ is bounded in the nonheating phase and unbounded in the heating phase. At the phase transition the spectrum of the effective Hamiltonian is continuous, as for the SSD Hamiltonian. The effective description of the heating phase is similar to one of the Mathieu oscillator for which the effective Hamiltonian in the unstable phase is not bounded from below and the phases are classified by a quadratic invariant of $S U(1,1)[31]$.
Note that in the heating case, the expression for the density of $\mathcal{H}_{\text {eff }}$ bears marked similarities to the one of an entanglement Hamiltonian of a subsystem of size $\left[x_{c}, L-x_{c}\right]$ [32]. Finally, it is straightforward to generalize this derivation to the non-time-reversal-symmetric case, for which the effective Hamiltonian has the general form $\mathcal{H}_{\text {eff }}=b L_{0}+a L_{1}+c L_{-1}$, and the different phases are still classified by the sign of the $S L(2, \mathbb{R})$ quadratic Casimir invariant,

$$
c^{(2)}=\left[\frac{L}{2 \pi i\left(T_{0}+T_{1}\right)} \ln (\eta)\right]^{2},
$$

as expected. Indeed in the nonheating phase $\ln (\eta)$ is imaginary and therefore $c^{(2)}>0$, in the heating phase $\ln (\eta)$ is real and therefore $c^{(2)}<0$, and at the phase transition $\eta=1$ and thus $c^{(2)}=0$.

\section{DISCUSSION}

The Floquet drive alternating between a uniform and a SSD CFT provides an exactly solvable nonequilibrium system with a rich phase diagram. We present exact analytical results describing the propagation of excitations as well as energy density in the system. Dynamics in the heating phase are understood by analogy to two black hole horizons and null geodesics in a curved but stationary spacetime geometry. Beyond the timescale fixed by the inverse Hawking temperature, excitations are absorbed by the black holes along with an accumulation of energy at these horizons. We demonstrate numerically that the CFT provides a surprisingly robust description of driven critical spin chains. Cold-atomic gases are a promising platform to realize such deformed Hamiltonians $[33,34]$, thereby opening up the possibility for experimental observation of emergent black hole dynamics in $(1+1)$ dimensional quantum systems.

Note added. We note that Ref. [35] has also discussed similar results pertaining to accumulation of energy at two points.

\section{ACKNOWLEDGMENTS}

We thank Xueda Wen, Koji Hashimoto, Shouvik Datta, and Alessandro Sfondrini for very helpful discussions. This project has received funding from the European Research Council (ERC) under the European Union's Horizon 2020 research and innovation program (ERC-StG-Neupert-757867PARATOP and Marie Sklodowska-Curie Grant Agreement No. 701647).

\section{APPENDIX A: DYNAMICAL TWO-POINT FUNCTION}

In this section we compute the dynamical two-point function defined as $F\left(x, t ; x_{0}, 0\right) \equiv\left\langle\phi(x, t) \phi\left(x_{0}, 0\right)\right\rangle$ corresponding to a primary field $\phi$ of conformal dimension $h$. The time evolution of the primary is governed by the Floquet Hamiltonian $\mathcal{H}_{\mathrm{F}}(t)$ defined in the main text. We closely follow the strategy employed in Ref. [8] wherein the time evolution of the entanglement entropy for a system driven by $\mathcal{H}_{\mathrm{F}}(t)$ was computed. Within this setup, we work in imaginary time $\tau$, and introduce Euclidean coordinates $\omega=\tau+i x$. Before getting to the computation for an $n$-cycle drive, we describe 
the 1-cycle drive as a warm-up. The two-point function is

$$
\begin{aligned}
F\left(x, \tau ; x_{0}, 0\right)= & \left\langle e^{\tau_{1} \mathcal{H}_{\mathrm{SSD}}} e^{\tau_{0} \mathcal{H}_{0}} \phi\left(\omega_{1}, \bar{\omega}_{1}\right) e^{-\tau_{0} \mathcal{H}_{0}} e^{-\tau_{1} \mathcal{H}_{\mathrm{SSD}}}\right. \\
& \left.\times \phi\left(\omega_{0}, \bar{\omega}_{0}\right)\right\rangle,
\end{aligned}
$$

where $\omega_{1}=0+i x, \omega_{0}=0+i x_{0}$ and $\tau=\tau_{0}+\tau_{1}$. $\mathcal{H}_{\mathrm{SSD}}$ and $\mathcal{H}_{0}$ are the SSD and uniform Hamiltonian described in the main text. Next, under the conformal mapping $z=\exp \left\{\frac{2 \pi \omega}{L}\right\}$, the two-point function transforms as

$$
\begin{aligned}
F\left(x, \tau ; x_{0}, 0\right)= & \left(\frac{2 \pi}{L}\right)^{4 h}\left\langle e^{\tau_{1} \mathcal{H}_{\mathrm{SSD}}} e^{\tau_{0} \mathcal{H}_{0}} \phi\left(z_{1}, \bar{z}_{1}\right) e^{-\tau_{0} \mathcal{H}_{0}}\right. \\
& \left.\times e^{-\tau_{1} \mathcal{H}_{\mathrm{SSD}}} \phi\left(z_{0}, \bar{z}_{0}\right)\right\rangle .
\end{aligned}
$$

To compute the time evolution with $\mathcal{H}_{\mathrm{SSD}}$ in the complex plane, we introduce the so-called Möbius Hamiltonian [17]

$$
\begin{aligned}
\mathcal{H}_{\text {Möb }(\theta)}= & L_{0}-\frac{\tanh (2 \theta)}{2}\left(L_{1}+L_{-1}\right)+\bar{L}_{0} \\
& -\frac{\tanh (2 \theta)}{2}\left(\bar{L}_{1}+\bar{L}_{-1}\right),
\end{aligned}
$$

defined for $\theta \in \mathbb{R}^{+}$. Interestingly, there exists an $S L(2, \mathbb{R})$ transformation mapping the Möbius Hamiltonian to a uniform Hamiltonian. Such mapping is explicitly given by

$$
\hat{z}=f(z)=\frac{-\cosh (\theta) z+\sinh (\theta)}{\sinh (\theta) z-\cosh (\theta)} .
$$

In the $\hat{z}$ coordinates, $\mathcal{H}_{\operatorname{Möb}(\theta)} \propto \frac{2 \pi}{L \cosh (2 \theta)}\left(L_{0}+\bar{L}_{0}\right)$. Thus the time evolution with $\mathcal{H}_{\mathrm{Möb}(\theta)}$ for a time $\tau$ in the $\hat{z}$ coordinates is a simple dilation by a factor $\lambda=\exp \left\{\frac{2 \pi \tau}{L \cosh 2 \theta}\right\}$. Then going back to the original coordinates, the whole time evolution with $\mathcal{H}_{\operatorname{Möb}(\theta)}$ amounts to a simple change of coordinates $z_{\theta}^{\text {new }}(z)=f^{-1}(\lambda f(z))$ (in the following text we often leave the $z$ dependence of the conformal mappings implicit):

$z_{\theta}^{\text {new }}(z)=\frac{[(1-\lambda) \cosh (2 \theta)-(\lambda+1)] z+(\lambda-1) \sinh (2 \theta)}{(1-\lambda) \sinh (2 \theta) z+[(\lambda-1) \cosh (2 \theta)-(\lambda+1)]}$.

The Hamiltonian $\mathcal{H}_{0}$ and $\mathcal{H}_{\mathrm{SSD}}$ can be seen as two different limits of the interpolating Hamiltonian $\mathcal{H}_{\mathrm{Möb}(\theta)}$. Indeed, $\mathcal{H}_{0}=\mathcal{H}_{\mathrm{Möb}(0)}$ and $\mathcal{H}_{\mathrm{SSD}}=\mathcal{H}_{\mathrm{Möb}(\theta \rightarrow \infty)}$. From this observation, it may be deduced that one can first evaluate $e^{\tau_{0} \mathcal{H}_{0}} \phi(\omega, \bar{\omega}) e^{-\tau_{0} \mathcal{H}_{0}}$ by applying the method in the case $\theta=0$ :

$$
\begin{aligned}
e^{\tau_{0} \mathcal{H}_{0}} \phi\left(\omega_{1}, \bar{\omega}_{1}\right) e^{-\tau_{0} \mathcal{H}_{0}}= & \left(\frac{2 \pi}{L}\right)^{2 h}\left[\left.\left.\frac{\partial z_{\theta=0}^{\text {new }}}{\partial z}\right|_{z_{1}} \frac{\partial \bar{z}_{\theta=0}^{\text {new }}}{\partial \bar{z}}\right|_{\bar{z}_{1}}\right]^{h} \\
& \times \phi\left(z_{\theta=0}^{\text {new }}\left(z_{1}\right), \bar{z}_{\theta=0}^{\text {new }}\left(z_{1}\right)\right) .
\end{aligned}
$$

By looking at the expression for $z_{\theta}^{\text {new }}(z)$ in Eq. (A5), we get $z_{\theta=0}^{\text {new }}(z)=\lambda z$, which is a dilatation in the $z$ plane, as expected for the uniform Hamiltonian $\mathcal{H}_{0}$. Next, we need to evaluate

$$
\begin{aligned}
& e^{\tau_{1} \mathcal{H}_{\mathrm{SSD}}}\left[e^{\tau_{0} \mathcal{H}_{0}} \phi\left(z_{1}, \bar{z}_{1}\right) e^{-\tau_{0} \mathcal{H}_{0}}\right] e^{-\tau_{1} \mathcal{H}_{\mathrm{SSD}}} \propto e^{\tau_{1} \mathcal{H}_{\mathrm{SSD}}} \phi\left(\lambda z_{1}, \lambda \bar{z}_{1}\right) \\
& \times e^{-\tau_{1} \mathcal{H}_{\mathrm{SSD}}},
\end{aligned}
$$

which can be obtained by using expression of $z_{\theta}^{\text {new }}$ in the limit $\theta \rightarrow \infty$. This just amounts to going to the coordinates $\tilde{z}_{1}$, defined as

$$
\tilde{z}_{1}=\lim _{\theta \rightarrow \infty} z_{\theta}^{\text {new }}(\lambda z)=\frac{\left(1+\frac{\pi \tau_{1}}{L}\right) e^{\frac{2 \pi \tau_{0}}{L}} z-\frac{\pi \tau_{1}}{L}}{\frac{\pi \tau_{1}}{L} e^{\frac{2 \pi \tau_{0}}{L}} z+\left(1-\frac{\pi \tau_{1}}{L}\right)} .
$$

Hence, $\tilde{z}_{1}$ is once again related to $z$ by a Möbius transformation, as expected because it is obtained via a composition of two (invertible) Möbius transformations. Consequently the time evolution $e^{\tau_{1} \mathcal{H}_{\text {SSD }}} e^{\tau_{0} \mathcal{H}_{0}} \phi(z, \bar{z}) e^{-\tau_{0} \mathcal{H}_{0}} e^{-\tau_{1} \mathcal{H}_{\text {SSD }}}$ for a 1 -cycle drive of any primary field of a CFT can be reduced to a normalized Möbius transformation

$$
\tilde{z}_{1}=\frac{a z+b}{c z+d}
$$

with

$$
\begin{gathered}
a=\left(1+\frac{\pi \tau_{1}}{L}\right) e^{\frac{\pi \tau_{0}}{L}}, \quad b=-\frac{\pi \tau_{1}}{L} e^{-\frac{\pi \tau_{0}}{L}}, \\
c=\frac{\pi \tau_{1}}{L} e^{\frac{\pi \tau_{0}}{L}}, \quad d=\left(1-\frac{\pi \tau_{1}}{L}\right) e^{-\frac{\pi \tau_{0}}{L}} .
\end{gathered}
$$

Explicitly the two-point function at different times for a 1cycle drive is

$$
\begin{aligned}
& \left\langle e^{\tau_{1} \mathcal{H}_{\mathrm{SSD}}} e^{\tau_{0} \mathcal{H}_{0}} \phi\left(\omega_{1}, \bar{\omega}_{1}\right) e^{-\tau_{0} \mathcal{H}_{0}} e^{-\tau_{1} \mathcal{H}_{\mathrm{SSD}}} \phi\left(\omega_{0}, \bar{\omega}_{0}\right)\right\rangle \\
& =\left(\frac{2 \pi}{L}\right)^{4 h}\left[\left.\left.\frac{\partial \tilde{z}_{1}}{\partial z}\right|_{z_{1}} \frac{\partial \overline{\tilde{z}}_{1}}{\partial \bar{z}}\right|_{\bar{z}_{1}}\right]^{h}\left\langle\phi\left(\tilde{z}_{1}, \tilde{z}_{1}\right) \phi\left(z_{0}, \bar{z}_{0}\right)\right\rangle .
\end{aligned}
$$

Therefore we learn that the time evolution of any primary field during one cycle of this Floquet drive between $\mathcal{H}_{0}$ and $\mathcal{H}_{\mathrm{SSD}}$ only amounts to a conformal transformation, as seen in (A10). The main task now is to find how to generalize this result to the full Floquet drive with $n$ cycles. Clearly, the $n$-cycle Floquet time evolution will just amount to a change of coordinates to $\tilde{z}_{n}$, defined as

$$
\tilde{z}_{n}(z)=\underbrace{\left(\tilde{z}_{1} \circ \ldots \circ \tilde{z}_{1}\right)}_{n \text { times }}(z) .
$$

This means that increasing the number of cycles only amounts to composing the 1-cycle transformation with itself.

The $n$-cycle Möbius transformation can be computed by writing the 1-cycle Möbius transformation in its so-called normal form. Introducing the two fixed-points $\gamma_{1}, \gamma_{2}$ and the multiplier $\eta$,

$$
\begin{aligned}
\gamma_{1} & =\frac{a-d-\sqrt{(a-d)^{2}+4 b c}}{2 c}, \\
\gamma_{2} & =\frac{a-d+\sqrt{(a-d)^{2}+4 b c}}{2 c}, \\
\eta & =\frac{a+d+\sqrt{(a-d)^{2}+4 b c}}{a+d-\sqrt{(a-d)^{2}+4 b c}} .
\end{aligned}
$$

The normal form of $\tilde{z}_{1}$ is then

$$
\frac{\tilde{z}_{1}-\gamma_{1}}{\tilde{z}_{1}-\gamma_{2}}=\eta \frac{z-\gamma_{1}}{z-\gamma_{2}} \text {. }
$$

It can be shown that in normal form the $n$-cycle evolution simply amounts to

$$
\frac{\tilde{z}_{n}-\gamma_{1}}{\tilde{z}_{n}-\gamma_{2}}=\eta^{n} \frac{z-\gamma_{1}}{z-\gamma_{2}} .
$$


Then all the stroboscopic time evolution is encoded in the Möbius multiplier $\eta$. This defines different phases, classified by the trace squared of the 1-cycle transformation [8]:

$$
\operatorname{Tr}^{2}\left(\begin{array}{ll}
a & b \\
c & d
\end{array}\right)=4(1-\Delta)
$$

Indeed if $\Delta>0$ the associated transformation is elliptic and $\eta$ is a phase: the system does not heat. If $\Delta<0$ the associated transformation is hyperbolic and $\eta$ is a positive number: the system heats. $\Delta=0$ corresponds to a parabolic Möbius transformation, $\eta=1$, and the system is at the phase transition. After analytic continuation, $\Delta$ is written as

$$
\Delta=\left[1-\left(\frac{\pi T_{1}}{L}\right)^{2}\right] \sin ^{2}\left(\frac{\pi T_{0}}{L}\right)+\frac{\pi T_{1}}{L} \sin \left(\frac{2 \pi T_{0}}{L}\right) .
$$

The $n$-cycle Möbius transformation can be explicitly written in terms of the parameters of the system as Eq. (A17),

$$
\tilde{z}_{n}=\frac{\mathfrak{a} z+\mathfrak{b}}{\mathfrak{c} z+\mathfrak{d}}
$$

with

$$
\begin{aligned}
& \mathfrak{a}=\gamma_{1}-\eta^{n} \gamma_{2}, \quad \mathfrak{b}=\left(\eta^{n}-1\right) \gamma_{1} \gamma_{2}, \\
& \mathfrak{c}=1-\eta^{n}, \quad \mathfrak{d}=\gamma_{1} \eta^{n}-\gamma_{2} .
\end{aligned}
$$

Then the stroboscopic time evolution $t=n\left(T_{0}+T_{1}\right)$ of any primary field $\phi$ can be computed by using this conformal transformation. We stress the fact that here the time evolution is stroboscopic in order to get an analytic handle on the long-time dynamics. However, by sacrificing some analytic succinctness we can actually access the full continuous time evolution. The two-point function at different times is directly obtained with Eq. (A18),

$$
\begin{aligned}
\left\langle\phi(x, t) \phi\left(x_{0}, 0\right)\right\rangle= & \left(\frac{2 \pi}{L}\right)^{4 h}\left[\left.\left.\frac{\partial \tilde{z}_{n}}{\partial z}\right|_{z_{1}} \frac{\partial \overline{\tilde{z}}_{n}}{\partial \bar{z}}\right|_{\bar{z}_{1}}\right]^{h} \\
& \times\left\langle\phi\left(\tilde{z}_{n}, \overline{\tilde{z}}_{n}\right) \phi\left(\tilde{z}_{0}, \overline{\tilde{z}}_{0}\right)\right\rangle .
\end{aligned}
$$

The correlator $\left\langle\phi\left(\tilde{z}_{n}, \overline{\tilde{z}}_{n}\right) \phi\left(\tilde{z}_{0}, \overline{\tilde{z}}_{0}\right)\right\rangle$ can either be computed within the ground state of $\mathcal{H}_{0}$ with open boundary con- ditions $|G\rangle$, or the $S L(2, \mathbb{C})$ invariant vacuum $|0\rangle$ of the periodic chain. As $|0\rangle$ is an eigenstate of $\mathcal{H}_{\mathrm{SSD}}$, the Floquet dynamics should be trivial when computing correlation functions at equal times, as the SSD time evolution is just a phase. However for dynamical two-point functions $\left\langle 0\left|e^{i \mathcal{H}_{\mathrm{SSD}} t} \phi(x, 0) e^{-i \mathcal{H}_{\mathrm{SSD}} t} \phi\left(x_{0}, 0\right)\right| 0\right\rangle$ the result should not be trivial as $|\Phi\rangle \equiv \phi\left(x_{0}, 0\right)|0\rangle$ is not an eigenstate of $\mathcal{H}_{\mathrm{SSD}}$ in general. Therefore this choice for the computation of $F\left(x, t ; x_{0}, 0\right)$ is legitimate. In the case of open boundary conditions, we need to use the mapping $z \rightarrow \sqrt{z}$ to map the complex plane with a slit to the upper half plane, and then evaluate the two-point function in the upper half plane [36]. This introduces some complications regarding branch cuts of the square-root mapping. For simplicity, we choose the periodic case, where

$$
\left\langle 0\left|\phi\left(\tilde{z}_{n}, \overline{\tilde{z}}_{n}\right) \phi\left(\tilde{z}_{0}, \overline{\tilde{z}}_{0}\right)\right| 0\right\rangle \propto \frac{1}{\left(z_{0}-\tilde{z}_{n}\right)^{2 h}} \frac{1}{\left(\bar{z}_{0}-\overline{\tilde{z}}_{n}\right)^{2 h}} .
$$

This leads to the final formula for the two-point function at different times for $n$ cycles:

$$
\begin{aligned}
\left\langle 0\left|\phi(x, t) \phi\left(x_{0}, 0\right)\right| 0\right\rangle= & \left(\frac{2 \pi}{L}\right)^{4 h}\left[\left.\left.\frac{\partial \tilde{z}_{n}}{\partial z}\right|_{z_{1}} \frac{\partial \tilde{z}_{n}}{\partial \bar{z}}\right|_{\bar{z}_{1}}\right]^{h} \\
& \times \frac{1}{\left(z_{0}-\tilde{z}_{n}\right)^{2 h}} \frac{1}{\left(\bar{z}_{0}-\overline{\tilde{z}}_{n}\right)^{2 h}} .
\end{aligned}
$$

It can further be shown that the derivative term simplifies to

$$
\left.\left.\frac{\partial \tilde{z}_{n}}{\partial z}\right|_{z_{1}} \frac{\partial \overline{\tilde{z}}_{n}}{\partial \bar{z}}\right|_{\bar{z}_{1}}=\frac{(\mathfrak{a} \mathfrak{d}-\mathfrak{b} \mathfrak{c})^{2}}{\left[\mathfrak{c}^{2}+\mathfrak{d}^{2}+2 \mathfrak{c} \mathfrak{d} \cos \left(\frac{2 \pi x}{L}\right)\right]^{2}}
$$

In the heating phase, $\eta$ is a real positive number, such that $\eta^{n}$ tends either to 0 or $\infty$ depending on the sign of $\eta-1$, corresponding to $\tilde{z}_{n}$ converging either to $\gamma_{1}$ or $\gamma_{2}$. Then $\left\langle\phi\left(\tilde{z}_{n}, \overline{\tilde{z}}_{n}\right) \phi\left(z_{0}, \bar{z}_{0}\right)\right\rangle$ tends to a constant, and the derivative term (A21) is exponentially suppressed for every $x \notin\left\{x_{c}, L-x_{c}\right\}$, with $x_{c}$ defined by the fixed points: $\gamma_{1 / 2}=e^{2 \pi x_{c} / L}$, where $\gamma_{1 / 2}$ corresponds to $\gamma_{2}$ if $\tilde{z}_{n}$ converges to $\gamma_{1}$, and vice versa. This can be seen explicitly in Eq. (A22),

$$
\left.\left.\frac{\partial \tilde{z}_{n}}{\partial z}\right|_{z_{1}} \frac{\partial \bar{z}_{n}}{\partial \bar{z}}\right|_{\bar{z}_{1}}=\frac{\left(\gamma_{1}-\gamma_{2}\right)^{4}}{\left\{\eta^{-n}\left(1+\gamma_{2}^{2}-2 \gamma_{2} \cos \frac{2 \pi x}{L}\right)+2\left[\left(\gamma_{1}+\gamma_{2}\right) \cos \frac{2 \pi x}{L}-1-\gamma_{1} \gamma_{2}\right]+\eta^{n}\left(1+\gamma_{1}^{2}-2 \gamma_{1} \cos \frac{2 \pi x}{L}\right)\right\}^{2}} .
$$

The expression (A22) has a two poles, either in $x_{c}=$ $\frac{L}{2 \pi} \arccos \left(\frac{1+\gamma_{2}^{2}}{2 \gamma_{2}}\right)$ and $L-x_{c}$ if $\lim _{n \rightarrow \infty} \eta^{n}=0$, or in $x_{c}=$ $\frac{L}{2 \pi} \arccos \left(\frac{1+\gamma_{1}^{2}}{2 \gamma_{1}}\right)$ and $L-x_{c}$ if $\lim _{n \rightarrow \infty} \eta^{n}=+\infty$. Therefore $F\left(x, t ; x_{0}, 0\right)$ remains finite even at very long times at these two points, for any choice of $x_{0}$. The interpretation is that even after a very long number of driving cycles, the excitations will always arrive at one of these two points at stroboscopic times. Therefore these particular points, only defined with the choice of $T_{0} / L$ and $T_{1} / L$, act as attractors for the excitations, which will be better understood within a stroboscopic black hole picture in an effective spacetime in the next section. In the nonheating phase, $\eta$ is a phase, and then after analytic continuation the periodicity reads $T_{E}=2 \pi \frac{\left(T_{0}+T_{1}\right)}{|\arg (\eta)|}$. The excitations are then propagating periodically with $T_{E}$. However if $T_{E} /\left(T_{0}+T_{1}\right) \notin \mathbb{Q}$, the system is pseudoperiodic, as the two-point function is only defined at stroboscopic times.

\section{APPENDIX B: EFFECTIVE CURVED SPACETIME}

In this section we derive the effective spacetime metric for the Floquet drive defined at stroboscopic times. We are interested in finding some coordinates $\tilde{z}_{n}$ in which the effective metric describing the $n$-cycle Floquet drive is conformally 
flat, and then going back to the original coordinates $(x, \tau)$ to get the expression of the metric. Such coordinates are called isothermal coordinates and always exist in $(1+1)$ dimensional spacetimes. For the Floquet drive, they are given by the effective Möbius transformation (A17), so that the metric reads

$$
d s^{2}=e^{-2 \sigma(x, \tau)} d \tilde{z}_{n} d \overline{\tilde{z}}_{n} .
$$

Introducing the real and imaginary parts of $\tilde{z}_{n}$,

$$
\begin{array}{r}
\tilde{u}_{n}(x, \tau)=\operatorname{Re}\left(\tilde{z}_{n}\right)=\frac{\mathfrak{a} \mathfrak{c}+\mathfrak{b} \mathfrak{d}+(\mathfrak{a} \mathfrak{d}+\mathfrak{b} \mathfrak{c}) \cos \left(\frac{2 \pi x}{L}\right)}{\mathfrak{c}^{2}+\mathfrak{d}^{2}+2 \mathfrak{c} \mathfrak{d} \cos \left(\frac{2 \pi x}{L}\right)}, \\
\tilde{v}_{n}(x, \tau)=\operatorname{Im}\left(\tilde{z}_{n}\right)=\frac{(\mathfrak{a} \mathfrak{d}-\mathfrak{b} \mathfrak{c}) \sin \left(\frac{2 \pi x}{L}\right)}{\mathfrak{c}^{2}+\mathfrak{d}^{2}+2 \mathfrak{c} \mathfrak{d} \cos \left(\frac{2 \pi x}{L}\right)} .
\end{array}
$$

The effective metric reads $e^{2 \sigma(x, \tau)} d s^{2}=d \tilde{u}_{n}^{2}+d \tilde{v}_{n}^{2}$. It is now straightforward to apply the change to $(x, \tau)$ coordinates. After some computations, the metric takes the familiar form, after analytic continuation:

$$
d \tilde{z}_{n} d \overline{\tilde{z}}_{n}=e^{2 \sigma(x, \tau)}\left[d x^{2}-g(x) d t^{2}+2 h(x) d x d t\right] .
$$

The values we find for $g(x)$ and $h(x)$ are then given by Eqs. (B3) and (B4):

$$
\begin{gathered}
g(x)=\zeta^{2} \prod_{i=1}^{2}\left[1+\gamma_{i}^{2}-2 \gamma_{i} \cos \left(\frac{2 \pi x}{L}\right)\right], \\
h(x)=i \zeta\left(\gamma_{1} \gamma_{2}-1\right) \sin \left(\frac{2 \pi x}{L}\right),
\end{gathered}
$$

where $\zeta=-\frac{L}{2 \pi i} \frac{1}{\left(T_{0}+T_{1}\right)} \frac{\ln (\eta)}{\left(\gamma_{1}-\gamma_{2}\right)}$, and as before $\eta$ is the multiplier of the Möbius transformation, which is a complex exponential in the nonheating phase and a real exponential in the heating phase, and $\gamma_{1}, \gamma_{2}$ are the two fixed points of the 1-cycle Möbius transformation. After analytic continuation, both $g(x)$ and $h(x)$ are real-valued functions.

The Weyl prefactor $e^{2 \sigma(x, \tau)}$ is a positive number before analytic continuation,

$$
e^{2 \sigma(x, \tau)}=\left.\left.\left(\frac{2 \pi}{L}\right)^{2} \frac{\partial \tilde{z}_{n}}{\partial z}\right|_{z_{1}} \frac{\partial \overline{\tilde{z}}_{n}}{\partial \bar{z}}\right|_{\bar{z}_{1}} .
$$

Inverting the Weyl transformation, the metric is finally given by

$$
d s^{2}=d x^{2}-g(x) d t^{2}+2 h(x) d x d t .
$$

In particular we note the following fact concerning isothermal coordinates: the correlation functions in curved-spacetime coordinates $(x, \tau)$ are related to correlation functions in conformally flat spacetime in the isothermal coordinates $\left(\tilde{u}_{n}, \tilde{v}_{n}\right)$ by the formula $[7,20]$

$$
\left\langle\phi(x, \tau) \phi\left(x_{0}, 0\right)\right\rangle=e^{2 h \sigma\left(x_{0}, 0\right)} e^{2 h \sigma(x, \tau)}\left\langle\phi\left(\tilde{z}_{n}, \overline{\tilde{z}}_{n}\right) \phi\left(\tilde{z}_{0}, \overline{\tilde{z}}_{0}\right)\right\rangle_{\text {flat }} .
$$

The correlation function $\left\langle\phi\left(\tilde{z}_{n}, \overline{\tilde{z}}_{n}\right) \phi\left(\tilde{z}_{0}, \overline{\tilde{z}}_{0}\right)\right\rangle_{\text {flat }}$ is just a correlation function evaluated in usual flat spacetime. In the case of periodic boundary conditions, this simplifies to

$$
\left\langle\phi\left(\tilde{z}_{n}, \overline{\tilde{z}}_{n}\right) \phi\left(\tilde{z}_{0}, \overline{\tilde{z}}_{0}\right)\right\rangle_{\text {flat }}=\frac{1}{\left(z_{0}-\tilde{z}_{n}\right)^{2 h}} \frac{1}{\left(\bar{z}_{0}-\overline{\tilde{z}}_{n}\right)^{2 h}} .
$$

Putting this equation together with (B5) in (B7), we obtain the formula (A20), from the curved-spacetime approach.

The null geodesics of this $(1+1)$-dimensional spacetime are uniquely determined by the condition $d s^{2}=0$. Thus they are the solutions of the equation $2 h(x(t)) \dot{x}(t)+\dot{x}^{2}(t)-$ $g(x(t))=0$ :

$$
\pm t(x)=\int_{x_{0}}^{x} d x^{\prime} \frac{1}{\sqrt{h\left(x^{\prime}\right)^{2}+g\left(x^{\prime}\right)} \mp h\left(x^{\prime}\right)} .
$$

Then the local group velocity of the excitations is $v(x)=$ $h(x) \mp \sqrt{h(x)^{2}+g(x)}$, where the sign corresponds to chiral and antichiral excitations. The expression (B6) is not timereversal invariant because of the off-diagonal term $h(x)$. Only if $\gamma_{1} \gamma_{2}=1, h(x)=0$ and the system is time-reversal invariant. This can be fulfilled by starting the drive in a symmetric way. For concreteness, we shift the origin of time by $\frac{T_{0}}{2}$ :

$$
\mathcal{H}_{\mathrm{F}}(t)= \begin{cases}\mathcal{H}_{0}, & 0<t<\frac{T_{0}}{2}, \\ \mathcal{H}_{\mathrm{SSD}}, & \frac{T_{0}}{2}<t<\frac{T_{0}}{2}+T_{1}, \\ \mathcal{H}_{0}, & \frac{T_{0}}{2}+T_{1}<t<\frac{3 T_{0}}{2}+T_{1}, \\ \text { etc. } & \end{cases}
$$

The associated 1-cycle Möbius transformation is therefore given by the equation

$$
\tilde{z}_{1}=\frac{\left(1+\frac{\pi \tau_{1}}{L}\right) e^{\frac{\pi \tau_{0}}{L}} z-\frac{\pi \tau_{1}}{L}}{\frac{\pi \tau_{1}}{L} z+\left(1-\frac{\pi \tau_{1}}{L}\right) e^{-\frac{\pi \tau_{0}}{L}}} .
$$

It is interesting to compare (B11) and (A9). The coefficients $a$ and $d$ are the same as in the nonsymmetric case, but not $b$ and $c$. Furthermore $b c$ is unchanged. Thus, looking at the definitions of the fixed points and the multiplier, this only redefines the denominators of $\gamma_{1}$ and $\gamma_{2}$, and keeps the multiplier $\eta$ invariant. It can then be shown that $\gamma_{1} \gamma_{2}=1$. Furthermore in the heating phase $\left|\gamma_{1}\right|=\left|\gamma_{2}\right|=1$, and therefore in the time-reversal symmetric situation $\gamma_{1}=\gamma_{2}^{*}$, whereas in the nonheating phase $\gamma_{1}$ and $\gamma_{2}$ are both real and inverse of each other. In this case, the metric is simplified to $d s^{2}=d x^{2}-$ $g(x) d t^{2}$. Applying the time-reversal condition, one finds that

$$
v(x)=[g(x)]^{1 / 2}=\frac{1}{2 \pi i} \frac{L \ln (\eta)}{\left(T_{0}+T_{1}\right)} \frac{\left(1+\gamma_{1}^{2}-2 \gamma_{1} \cos \frac{2 \pi x}{L}\right)}{\gamma_{1}^{2}-1} .
$$

This is the effective velocity of the excitations. In the heating phase their local group velocity goes to 0 at two points, which are found to be $x_{c}=\frac{L}{2 \pi} \arccos \left(\frac{1+\gamma_{1}^{2}}{2 \gamma_{1}}\right)$ and $L-$ $x_{c}$. After analytic continuation, it can be shown that $x_{c}=$ $\frac{L}{2 \pi} \arccos \left(\cos \frac{\pi T_{0}}{L}+\frac{L}{\pi T_{1}} \sin \frac{\pi T_{0}}{L}\right)$. Thus $x_{c} \in\left[0, \frac{L}{2}\right]$ in the heating phase, and $x_{c}$ is a complex number in the nonheating phase; therefore the velocity never goes to 0 . Thus in the heating phase at these two points, the velocity of the excitations vanishes, meaning that their world lines, following null geodesics of the metric, will tend to one of these two points. We rewrite the metric in the heating phase in terms of the horizon $x_{c}$. We first notice that as $\cos \left(\frac{2 \pi x_{c}}{L}\right)=\frac{1}{2} \frac{\gamma_{1}^{2}+1}{\gamma_{1}}$, then the effective deformation is rewritten directly in terms of the horizon

$$
v(x)=A\left(1-\frac{\cos \left(\frac{2 \pi x}{L}\right)}{\cos \left(\frac{2 \pi x_{c}}{L}\right)}\right),
$$


with $A=\frac{1+\gamma_{1}^{2}}{\gamma_{1}^{2}-1} \frac{1}{2 \pi i\left(T_{0}+T_{1}\right)} L \ln (\eta)$. Using trigonometric formulas, this leads to the desired form of the velocity

$$
v(x)=2 A \frac{\sin \left[\frac{\pi}{L}\left(x-x_{c}\right)\right] \sin \left[\frac{\pi}{L}\left(x+x_{c}\right)\right]}{\cos \left(\frac{2 \pi x_{c}}{L}\right)} .
$$

The effective metric can now be easily expressed in terms of the horizon $x_{c}$ :

$$
d s^{2}=-4 A^{2} \frac{\sin ^{2}\left[\frac{\pi}{L}\left(x-x_{c}\right)\right] \sin ^{2}\left[\frac{\pi}{L}\left(x+x_{c}\right)\right]}{\cos ^{2}\left(\frac{2 \pi x_{c}}{L}\right)} d t^{2}+d x^{2} .
$$

This form is still hard to interpret in terms of black hole metric. However, by doing an expansion around one of the two horizons, i.e., around $x_{c}$ or $L-x_{c}$, and keeping only the lowest-order contribution, only the contribution from one of the horizons should matter and the metric should be simpler. Therefore we expand the expression (B15) around $x_{c}$. At leading order in $x-x_{c}$, we may simplify:

$$
\begin{aligned}
\sin ^{2} & {\left[\frac{\pi}{L}\left(x-x_{c}\right)\right] \sin ^{2}\left[\frac{\pi}{L}\left(x+x_{c}\right)\right] } \\
& \approx \frac{\pi^{2}}{L^{2}}\left(x-x_{c}\right)^{2} \sin ^{2}\left(\frac{2 \pi x_{c}}{L}\right)+O\left(\left(x-x_{c}\right)^{3}\right) .
\end{aligned}
$$

The metric finally simplifies to

$$
d s^{2}=-4 A^{2} \tan ^{2}\left(\frac{2 \pi x_{c}}{L}\right) \frac{\pi^{2}}{L^{2}}\left(x-x_{c}\right)^{2} d t^{2}+d x^{2} .
$$

This metric is known as the Rindler metric, which describes an accelerated frame transformation of the flat Minkowski metric. Writing $C^{2}=4 A^{2} \tan ^{2}\left(\frac{2 \pi x_{c}}{L}\right) \frac{\pi^{2}}{L^{2}}$, the metric reads $d s^{2}=$ $-C^{2}\left(x-x_{c}\right)^{2} d t^{2}+d x^{2}$. One can now introduce the following coordinate change: $\frac{C}{2}\left(x-x_{c}\right)^{2}=\left(y-x_{c}\right)$. In the new coordinates, the metric reads

$$
d s^{2}=-2 C\left(y-x_{c}\right) d t^{2}+\frac{1}{2 C} \frac{1}{\left(y-x_{c}\right)} d y^{2} .
$$

This is a particular form of a black hole metric in $(1+1)$ dimensions [21-23]. Thus expanding our spacetime effective metric around one of the two horizons gives (at leading order) a black hole metric containing one horizon at $x=x_{c}$. One can also do that for the second horizon by expanding the metric around $L-x_{c}$, to get similar results: $d s^{2}=$ $-2 C\left[y-\left(L-x_{c}\right)\right] d t^{2}+\frac{1}{2 c} \frac{1}{\left[y-\left(L-x_{c}\right)\right]} d y^{2}$.

The Hawking temperature $\Theta_{\mathrm{H}}$ can be directly read-off from the expression of the metric as $\Theta_{\mathrm{H}}=\frac{|C|}{2 \pi}$. We can finally use the formula $\tan \left(\frac{2 \pi x_{c}}{L}\right)=\frac{1}{i} \frac{\gamma_{1}^{2}-1}{\gamma_{1}^{2}+1}$ to conclude that the Hawking temperature is given by $\Theta_{\mathrm{H}}=\frac{|\ln (\eta)|}{2 \pi\left(T_{0}+T_{1}\right)}$.

\section{APPENDIX C: EFFECTIVE HAMILTONIAN}

Using the effective metric in the time-reversal-symmetric case, we deduce that the stroboscopic effective Hamiltonian is $\mathcal{H}_{\text {eff }}=\int_{0}^{L} v(x) T_{00}(x) d x$. Using the Fourier decomposition of $v(x)$, given by Eq. (B12), and using the definition of the Virasoro generators $L_{n}=\frac{1}{2 \pi i} \oint d z z^{n+1} T(z)$, we can conclude that the stroboscopic Hamiltonian is

$$
\mathcal{H}_{\mathrm{eff}}=\alpha\left[L_{0}-\frac{\beta}{2}\left(L_{1}+L_{-1}\right)+\bar{L}_{0}-\frac{\beta}{2}\left(\bar{L}_{1}+\bar{L}_{-1}\right)\right],
$$

where $\alpha=\frac{1+\gamma_{1}^{2}}{\gamma_{1}^{2}-1} \frac{L}{2 \pi i\left(T_{0}+T_{1}\right)} \ln (\eta), \beta=\frac{2 \gamma_{1}}{1+\gamma_{1}^{2}}$, which are real numbers. It can further be shown using the expressions of the fixed points that $\beta^{-1}=\cos \left(\frac{\pi T_{0}}{L}\right)+\frac{L}{\pi T} \sin \left(\frac{\pi T_{0}}{L}\right)$. Therefore in the heating phase $|\beta|>1$, and in the nonheating phase $|\beta|<1$.

In the case $|\beta|<1$, the effective Hamiltonian is simply the Möbius Hamiltonian (A3). This observation is consistent with the fact that $F\left(x, t ; x_{0}, 0\right)$ is periodic in the nonheating phase. Indeed, the propagation of the excitations after a quench with the Möbius Hamiltonian is also periodic, with period $T=$ $\frac{1}{L \cosh (2 \theta)}$. Therefore the effective stroboscopic Hamiltonian in the nonheating phase in the time-reversal-symmetric case is just an interpolating Hamiltonian between $\mathcal{H}_{0}$ and $\mathcal{H}_{\text {SSD }}$. $\mathcal{H}_{\text {eff }}$ can be further written as the convex combination of the two original Hamiltonians:

$$
\mathcal{H}_{\text {eff }}=\alpha\left[(1-\beta) \mathcal{H}_{0}+\beta \mathcal{H}_{\mathrm{SSD}}\right] .
$$

Therefore, for $0<\beta<1$, the effective Hamiltonian interpolates between the uniform and the SSD Hamiltonian, as we already understood through the comparison with the Möbius Hamiltonian.

For $\beta>1$, the effective Hamiltonian cannot be understood as an interpolation between the two original Hamiltonians, giving rise to the physics of heating. The effective Hamiltonian in the heating phase can be rewritten, using (B14), as

$$
\mathcal{H}_{\mathrm{eff}}=2 L \Theta_{\mathrm{H}} \int_{0}^{L} d x \frac{\sin \left[\frac{\pi}{L}\left(x-x_{c}\right)\right] \sin \left[\frac{\pi}{L}\left(x+x_{c}\right)\right]}{\sin \left(\frac{2 \pi x_{c}}{L}\right)} T_{00}(x) .
$$

This form of the effective Hamiltonian is reminiscent of the entanglement Hamiltonian $K_{A}$ for a system of finite size [0, $\left.L\right]$, introduced in Ref. [32], with subsystem $A=\left(x_{c}, L-x_{c}\right)$. However here the Hamiltonian density is integrated over the whole chain. For such entanglement Hamiltonian, an effective local temperature can be defined, diverging at $x_{c}$ and $L-x_{c}$. This is an indication that energy should be absorbed exponentially at these two points.

For completeness, we also give the stroboscopic effective Hamiltonian in the non-time-reversal Floquet drive. In this case the velocity of the excitations is $v(x)=h(x) \mp$ $\sqrt{h(x)^{2}+g(x)}$, which has the following Fourier decomposition:

$$
\begin{aligned}
v(x)= & \zeta\left[\gamma_{1}+\gamma_{2}-\left(1+\gamma_{1} \gamma_{2}\right) \cos \left(\frac{2 \pi x}{L}\right)\right. \\
& \left. \pm i\left(-1+\gamma_{1} \gamma_{2}\right) \sin \left(\frac{2 \pi x}{L}\right)\right] .
\end{aligned}
$$

We therefore conclude that the general effective Hamiltonian has the form

$$
\begin{aligned}
\mathcal{H}_{\mathrm{eff}}= & \zeta\left[\left(\gamma_{1}+\gamma_{2}\right) L_{0}-\frac{1}{2}\left(\gamma_{1} \gamma_{2}+1\right)\left(L_{1}+L_{-1}\right)\right. \\
& \left.+\frac{1}{2}\left(\gamma_{1} \gamma_{2}-1\right)\left(L_{1}-L_{-1}\right)\right]=: b L_{0}+a L_{1}+c L_{-1},
\end{aligned}
$$


where we omitted the anti-holomorphic part. This is still a combination of $S L(2, \mathbb{R})$ generators as expected. It turns out that the effective Hamiltonian in this general case is classified by a quadratic Casimir invariant:

$$
c^{(2)}=b^{2}-4 a c=\left[\frac{L}{2 \pi i\left(T_{0}+T_{1}\right)} \ln (\eta)\right]^{2} .
$$

In the nonheating phase $\ln (\eta)$ is imaginary and therefore $c^{(2)}>0$, in the heating phase $\ln (\eta)$ is real and therefore $c^{(2)}<$ 0 , and at the phase transition $\eta=1$ and thus $c^{(2)}=0$.

This invariant has been introduced to characterize the three different classes of coadjoint orbits of $S L(2, \mathbb{R})$ [28]. In particular, the class of orbit with $c^{(2)}>0$ corresponds to the Hamiltonian possessing a discrete spectrum, and describes the nonheating phase. A representative of this class is the uniform Hamiltonian $\mathcal{H}_{0}$. The phase transition happens whenever $c^{(2)}=0$, which is the "light cone" of the coadjoint orbits of $\operatorname{SL}(2, \mathbb{R})$. The associated Hamiltonian has a continuous spectrum, and a representative of this class is the SSD Hamiltonian $\mathcal{H}_{\mathrm{SSD}}$. Finally the last class of orbits is characterized by $c^{(2)}<0$, and the spectrum of the associated Hamiltonian is unbounded from below. They correspond to the heating phase, and a typical representative of this class is the operator $L_{1}+L_{-1}[30]$.

\section{APPENDIX D: ENERGY DENSITY}

The different phases arising within the Floquet CFT were understood in Ref. [8] by computing the entanglement entropy, which grows linearly in the heating phase and oscillates with period $T_{E}$ in the nonheating phase. We would like to characterize more precisely these phases by computing the evolution of the energy density $\mathcal{E}(x, t)$ in the system. In particular, we expect to observe an exponential increase of energy in the heating phase precisely at the location of the two horizons $x_{c}$ and $L-x_{c}$, whereas the rest of the system should not absorb energy, to agree with our stroboscopic black hole picture. The energy density $\mathcal{E}(x, t)$ under the Floquet drive is defined by

$$
\mathcal{E}(x, t)=\left\langle\psi(t)\left|T_{00}(x)\right| \psi(t)\right\rangle .
$$

As usual $T_{00}$ is the energy density of the uniform CFT. $|\psi(t)\rangle=U(t)|G\rangle$ is the time-evolved ground state of the uniform Hamiltonian $H_{0}$, with open boundary conditions.
We chose open boundary conditions in this case, as in the periodic case $\mathcal{E}(x, t)=0$. In Euclidean coordinates, $T_{00}=$ $T(\omega)+\bar{T}(\bar{\omega})$. The strategy is the same as for the two-point function at different times: the first step is to map the strip to the complex plane with a slit, using the exponential mapping

$$
\begin{aligned}
& \left\langle G\left|e^{\tau \mathcal{H}_{\mathrm{SSD}}} T(\omega) e^{-\tau \mathcal{H}_{\mathrm{SSD}}}\right| G\right\rangle \\
& =\left(\frac{\partial z}{\partial \omega}\right)^{2}\left\langle G\left|e^{\tau \mathcal{H}_{\mathrm{SSD}}} T(z) e^{-\tau \mathcal{H}_{\mathrm{SSD}}}\right| G\right\rangle-\frac{\pi^{2} c}{6 L^{2}} .
\end{aligned}
$$

Then, the usual procedure consists in mapping the complex plane to itself in the Möbius $\tilde{z}_{n}$ coordinates, applying the time evolution, and transforming back to the $z$ coordinates. The extra terms coming from the Schwarzian derivative vanish because of $S L(2, \mathbb{R})$ invariance

$$
\begin{aligned}
& \left\langle G\left|e^{\tau \mathcal{H}_{\mathrm{SSD}}} T(\omega) e^{-\tau \mathcal{H}_{\mathrm{SSD}}}\right| G\right\rangle \\
& =\left(\frac{\partial z}{\partial \omega}\right)^{2}\left(\frac{\partial \tilde{z}_{n}}{\partial z}\right)^{2}\left\langle G\left|T\left(\tilde{z}_{n}\right)\right| G\right\rangle-\frac{\pi^{2} c}{6 L^{2}} .
\end{aligned}
$$

The final step is to evaluate the correlation function $\left\langle G\left|T\left(\tilde{z}_{n}\right)\right| G\right\rangle$ in a boundary CFT, defined on the complex plane with a slit on the real positive axis. This can be done using a square-root mapping $\sqrt{z}$ to the upper half plane $\mathbb{H}$. This gives a nontrivial Schwarzian derivative term given by $\{z, \sqrt{z}\}=$ $\frac{3}{8 z^{2}}$. The upper half plane can be mapped to the unit disk with a Möbius transformation; therefore, due to rotational symmetry, $\left\langle G\left|T\left(\sqrt{\tilde{z}_{n}}\right)\right| G\right\rangle_{\mathbb{H}}=0$ [37]. Finally, only the Schwarzian derivative term of the square-root mapping contributes to the energy density, which before analytic continuation reads

$$
\begin{aligned}
\mathcal{E}(x, t)= & \frac{c}{32}\left[\left(\frac{\partial z}{\partial \omega}\right)^{2}\left(\frac{\partial \tilde{z}_{n}}{\partial z}\right)^{2} \frac{1}{\tilde{z}_{n}^{2}}+\left(\frac{\partial \bar{z}}{\partial \bar{\omega}}\right)^{2}\left(\frac{\partial \overline{\tilde{z}}_{n}}{\partial \bar{z}}\right)^{2} \frac{1}{\overline{\tilde{z}}_{n}^{2}}\right] \\
& -\frac{\pi^{2} c}{3 L^{2}},
\end{aligned}
$$

for stroboscopic times $t=n\left(T_{0}+T_{1}\right)$. In the heating phase, as $\mathcal{E}\left(x_{c}, t\right) \sim \eta^{-2 n}$ at long time because of the derivative term $\frac{\partial \bar{z}_{n}}{\partial \bar{z}}$, we conclude that $\mathcal{E}\left(x_{c}, t\right) \sim e^{4 \pi \Theta_{\mathrm{H}} t}$, such that the Hawking temperature is really the heating rate. This is similar for the other horizon $L-x_{c}$, where the energy is also growing exponentially because of the other derivative term $\frac{\partial \tilde{z}_{n}}{\partial z}$. Therefore the energy density grows exponentially in the heating phase only at the positions of the two black holes, as expected. In the nonheating phase, the energy density oscillates in time with period $T_{E}=2 \pi \frac{\left(T_{0}+T_{1}\right)}{|\arg (\eta)|}$.
[1] Y. Tang, W. Kao, K.-Y. Li, S. Seo, K. Mallayya, M. Rigol, S. Gopalakrishnan, and B. L. Lev, Phys. Rev. X 8, 021030 (2018).

[2] M. Reitter, J. Näger, K. Wintersperger, C. Sträter, I. Bloch, A. Eckardt, and U. Schneider, Phys. Rev. Lett. 119, 200402 (2017).

[3] L. D'Alessio and M. Rigol, Phys. Rev. X 4, 041048 (2014).

[4] A. Lazarides, A. Das, and R. Moessner, Phys. Rev. E 90, 012110 (2014).

[5] A. Lazarides, A. Das, and R. Moessner, Phys. Rev. Lett. 112, 150401 (2014).

[6] S. Kinoshita, K. Murata, and T. Oka, J. High Energy Phys. 06 (2018) 096.
[7] X. Wen and J.-Q. Wu, Phys. Rev. B 97, 184309 (2018).

[8] X. Wen and J.-Q. Wu, arXiv:1805.00031.

[9] W. Berdanier, M. Kolodrubetz, R. Vasseur, and J. E. Moore, Phys. Rev. Lett. 118, 260602 (2017).

[10] A. Gendiar, R. Krcmar, and T. Nishino, Prog. Theor. Phys. 122, 953 (2009).

[11] T. Hikihara and T. Nishino, Phys. Rev. B 83, 060414(R) (2011).

[12] I. Maruyama, H. Katsura, and T. Hikihara, Phys. Rev. B 84, 165132 (2011).

[13] H. Katsura, J. Phys. A: Math. Theor. 45, 115003 (2012). 
[14] N. Allegra, J. Dubail, J.-M. Stéphan, and J. Viti, J. Stat. Mech. (2016) 053108.

[15] J. Dubail, J.-M. Stéphan, and P. Calabrese, SciPost Phys. 3, 019 (2017).

[16] N. Ishibashi and T. Tada, J. Phys. A: Math. Theor. 48, 315402 (2015).

[17] K. Okunishi, Prog. Theor. Exp. Phys. 2016, 063A02 (2016).

[18] X. Wen, S. Ryu, and A. W. W. Ludwig, Phys. Rev. B 93, 235119 (2016).

[19] S. Tamura and H. Katsura, Prog. Theor. Exp. Phys. 2017, 113A01 (2017).

[20] P. Ruggiero, Y. Brun, and J. Dubail, SciPost Phys. 6, 51 (2019).

[21] R. Mann, A. Shiekh, and L. Tarasov, Nucl. Phys. B 341, 134 (1990).

[22] J. D. Brown, M. Henneaux, and C. Teitelboim, Phys. Rev. D 33, 319 (1986)

[23] R. B. Mann, S. M. Morsink, A. E. Sikkema, and T. G. Steele, Phys. Rev. D 43, 3948 (1991).
[24] R. E. Goldstein, Phys. Today 71(9), 32 (2018).

[25] R. Chitra and O. Zilberberg, Phys. Rev. A 92, 023815 (2015).

[26] ITensor Library (Version 2.0.11), http://itensor.org.

[27] K. Choo, B. Lapierre, C. Tauber, A. Tiwari, T. Neupert, and R. Chitra, Sine-squared deformed Floquet dynamics for quantum spin chains near criticality (unpublished).

[28] E. Witten, Commun. Math. Phys. 114, 1 (1988).

[29] T. Tada, Prog. Theor. Exp. Phys. 2018, $061 \mathrm{~B} 01$ (2018).

[30] T. Tada, arXiv:1904.12414.

[31] V. Gritsev and A. Polkovnikov, SciPost Phys. 2, 021 (2017).

[32] J. Cardy and E. Tonni, J. Stat. Mech. (2016) 123103.

[33] C. Gross and I. Bloch, Science 357, 995 (2017).

[34] V. Borish, O. Marković, J. A. Hines, S. V. Rajagopal, and M. Schleier-Smith, Phys. Rev. Lett. 124, 063601 (2020).

[35] R. Fan, Y. Gu, A. Vishwanath, and X. Wen, arXiv:1908.05289.

[36] P. Calabrese and J. Cardy, J. Stat. Mech. (2007) P06008.

[37] P. Calabrese and J. Cardy, J. Phys. A 42, 504005 (2009). 